\title{
Comments on "A Gibbs sampler for a class of random convex polytopes"
}

\author{
Kentaro Hoffman, Jan Hannig and Kai Zhang \\ University of North Carolina at Chapel Hill
}

May 10, 2021

\begin{abstract}
In this comment we discuss relative strengths and weaknesses of simplex and Dirichlet Dempster-Shafer inference as applied to multi-resolution tests of independence.
\end{abstract}

\section{Introduction}

We would like to congratulate the authors for their contribution to this longstanding open problem in mathematical statistics. Their clever implementation of MCMC to obtain simplex based DempsterShafer (DS) samples for parameters of multinomial distribution and its connection to graph theory is extremely thought provoking. We expect that the contributions seen in this paper will have impacts for years to come.

In our comment, we would like to contribute our thoughts particularly on the applications of the various DS approaches to nonparametric tests of independence. Nonparametric dependence detection is a classical statistical problem but recently gains great interest from both statisticians and computer scientists due to its applications in machine learning. See Hoeffding (1948); Székely et al. (2007); Gretton et al. (2007); Reshef et al. (2011); Heller et al. (2013); Chatterjee (2020); Shi et al. (2020), and references therein.

One general approach in nonparametric tests of independence is the multi-resolution approach. See some recent work by Ma and Mao (2019); Zhang (2019); Lee et al. (2019); Gorsky and Ma (2018); Zhang et al. (2021). Some advantages of this approach include uniform consistency, minimax optimal power, clear interpretability and efficient computation.

The multi-resolution approach reduces the test of independence to the test of discrete uniformity over multinomial distributions. In this paper, we study the performance of such tests, particularly when the sample size is small compared to the resolution. We show potential gains in power and computational scalability from using an alternative Dirichlet DS method based on unpublished manuscript of Lawrence et al. (2009).

\section{Simplex and Dirichlet DS for Multinomial Data}

A crucial part to multi-resolution tests of independence is being able to conduct inference for parameters $\mathbf{p}=\left(p_{1}, \ldots p_{k}\right) \in \mathcal{P}=\left\{\mathbf{p}: 0 \leq p_{i}, \sum_{i=1}^{k} p_{i}=1\right\}$ using observation $\mathbf{z}=\left(z_{1}, \ldots z_{k}\right)$ 
following multinomial $(n, \mathbf{p})$ distribution. In particular, we will compare two different DS approaches to inference parameters $\mathbf{p}$.

The first, which we shall refer to as simplex $D S$, refers to the method used in the discussed paper. This version of the DS is based on inverting a very natural data generating algorithm proposed by Dempster et al. (1966) and described in detail in Section 2 of the discussed paper. In particular, a sample from the multinomial distribution is generated by sampling ancillary variables uniformly at random on a simplex and selecting the multinomial category depending on which section of the simplex the ancillary variable falls into, see Figure 1a in the discussed paper. To generate DS samples one needs to invert this data generating algorithm, i.e., sample new ancillary variables uniformly and find partitions of the simplex, determining the parameters $\mathbf{p}$, that would reproduces the observed data. The main challenge is that such partitions of the simplex exist only if the ancillary random variables fall into a rather complicated small polytope. The discussed paper proposes an ingenious Gibbs sampler that does just that. Once such ancillary variable $u^{(t)}$ is sampled, a linear programming problems need to be solved to obtain the particle $\mathcal{F}\left(u^{(t)}\right)$ of parameters $\mathbf{p}$ that are needed for inference.

While this Gibbs sampler offers a theoretically interesting representation using shortest path algorithm on graphs, we find that this algorithm has some drawbacks in terms of scalability. For example, generating 1000 MCMC samples a 7-Dimensional multinomial takes approximately 1 minute. However, converting the Gibbs sampler's results $u^{(t)}$ into a convex polytope $\mathcal{F}\left(u^{(t)}\right)$ takes nearly 20 times as long as running the Gibbs sampler. This is due to the large number of vertexes in the polytopes that result from the Simplex method. The simulation resulted in polygons with an average of 62 and a max over 250 edges. This places a large computational burden on the simplex method.

\begin{tabular}{|c|c|}
\hline Time Running the Gibbs Sampler & Time converting MCMC to convex polytope \\
\hline 2.6 seconds & 53.6 seconds \\
\hline
\end{tabular}

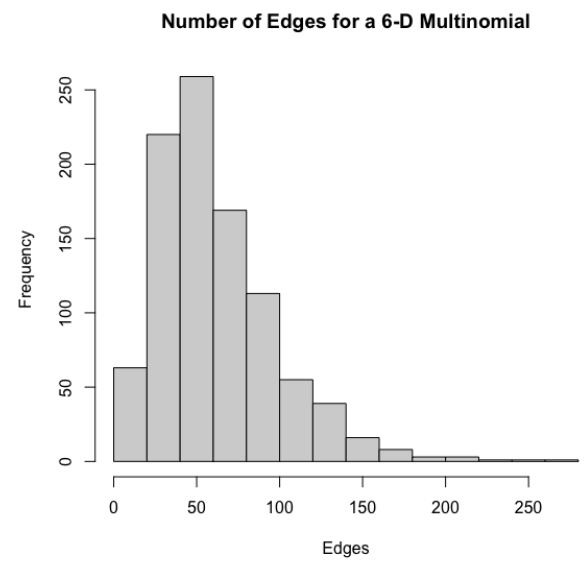

Figure 1: (Top): Runtime comparison of Gibbs sampler vs convex polytope computation. (Bottom): The number of edges in 1000 Convex polytopes from the Simplex method.

The other DS method was proposed in an unpublished manuscript of Lawrence et al. (2009) and we will be referring to as the Dirichlet DS. While Lawrence et al. (2009) obtain Dirchlet DS 
using a clever use of Dempster's rule of combination starting from $k$ binomial DSs. In particular, let $\mathbf{W}^{\star}$ follow Dirichlet $\left(1, z_{1}, \ldots, z_{k}\right)$ distribution and the Dirchlet DS polytope

$$
\mathcal{F}\left(\mathbf{W}^{\star}\right)=\left\{\mathbf{p}: W_{i}^{\star} \leq p_{i}, i=1, \ldots, k\right\}
$$

is a simplex with vertexes $\left(W_{1}^{\star}, W_{2}^{\star}, \ldots, W_{k}^{\star}\right),\left(W_{1}^{\star}+W_{0}^{\star}, W_{2}^{\star}, \ldots, W_{k}^{\star}\right),\left(W_{1}^{\star}, W_{2}^{\star}+W_{0}^{\star}, \ldots, W_{k}^{\star}\right), \ldots$, $\left(W_{1}^{\star}, W_{2}^{\star}, \ldots, W_{k}^{\star}+W_{0}^{\star}\right)$ (Hannig et al., 2016).

Dirichlet DS has a number of clear computational advantages. First, a independent sampling from Dirichlet $\left(1, z_{1}, \ldots, z_{k}\right)$ can be done directly using methods built into most software packages. Additionally the Dirichlet DS polytope is always a simplex, so for $k=7$ it yields a convex polytope with 8 vertexes, instead of a maximum of 250. This makes the Dirichlet DS to scale to much larger problems, e.g. multinomial distributions with thousands of categories, which is important for testing independence at high dimensions and/or high resolutions.

Next, many estimators of the multinomial proportions have the following invariance property: If we merge two categories, the estimate of the merged proportion is the sum of the proportions that are being merged. Investigation of (2.1) reveals that Dirichlet DS has this invariance property. Consequently, inference on proportions for categories in which we have observations is not influenced by addition or deletion of empty categories. As seen in Section 4.1 of the discussed paper, simplex DS does not have this invariance property, but whether this invariance property is desirable could be a matter of opinion.

Finally, let us assume that there were originally $n+r$ of the multinoulli experiments but we were only given results of $n$ and the other $r$ are missing. Notice that we are not assuming that these $r$ multinoullis are missing at random, in fact they could be deleted deliberately. The Dirichlet DS naturally accommodates this additional information by changing the distribution of $\mathbf{W}^{\star}$ to Dirichlet $\left(1+r, z_{1}, \ldots, z_{k}\right)$ consequently enlarging the polytope in (2.1). Such an enlargement of the DS polytopes is sometimes called weakening. It is not clear to us if simplex DS could be weakened to accommodate missing observations.

\section{DS Test of Independence}

For a pair of continuous random variables $(X, Y)$ distributed on $[0,1]^{2}$ we would like to test using $n$ i.i.d. samples $\left(X_{1}, Y_{1}\right) \ldots\left(X_{n}, Y_{n}\right)$ if $X$ is independent of $Y$. An important step towards this goal is to be able to test if $(X, Y)$ is uniform, or alternatively at what resolutions we have sufficient power to make a rejection. We adopt the notation of Gorsky and Ma (2018). For a joint sample space $\Omega=\Omega_{x} \times \Omega_{y}$ it is possible to create a coarse-to-fine discretization of $\Omega$. At resolution $k$, we partition $\Omega$ into sets as follows:

$$
\Omega=\bigcup_{i=1}^{k} \bigcup_{j=1}^{k} I_{i}^{k} \times I_{j}^{k}
$$

Where $i \in\{1, k\} I_{i}^{k}=\left[\frac{i-1}{k}, \frac{i}{k}\right)$. At the coarsest level, resolution $2, \Omega$ is discretized into four different pieces: $\Omega_{11}=\left[0, \frac{1}{2}\right] \times\left[0, \frac{1}{2}\right], \Omega_{12}=\left[0, \frac{1}{2}\right] \times\left(\frac{1}{2}, 1\right], \Omega_{21}=\left(\frac{1}{2}, 1\right] \times\left[0, \frac{1}{2}\right], \Omega_{22}=\left(\frac{1}{2}, 1\right] \times\left(\frac{1}{2}, 1\right]$ with

$$
\Omega=\Omega_{11} \cup \Omega_{21} \cup \Omega_{12} \cup \Omega_{22}
$$

These sets $\left\{I_{i}^{k} \times I_{j}^{k}\right\}$ each define statistics

$$
Z_{i, j}=\left|\left(X_{i}, Y_{j}\right) \in \Omega_{i, j}\right|
$$


which naturally describe a $k \times k$ contingency table. Under the classical multinomial sampling scheme for $k \times k$ contingency table, the statistics $Z_{i, j}$ are distributed:

$$
\left(Z_{11} \ldots Z_{k k} \mid \sum_{i, j} Z_{i, j}\right) \sim \operatorname{multinomial}\left(n,\left(p_{11}, \ldots p_{k k}\right)\right)
$$

To test if $(X, Y)$ are uniform at resolution $\mathrm{k}$, it suffices to discretize $\left(X_{1}, Y_{1}\right) \ldots\left(X_{n}, Y_{n}\right)$ into a $k \times k$ contingency table and used the counts $z_{i j}$ in each bin to obtain the distribution of random polytope $\Delta \subset \mathcal{P}=\left\{\mathbf{p}: \quad 0 \leq p_{i j}, \sum_{i=1}^{k} \sum_{j=1}^{k} p_{i j}=1\right\}$ following either simplex or Dirichlet DS distribution. The null hypothesis of uniformity should be rejected at this resolution if the null-hypothesis point $\left(k^{-2}, \ldots, k^{-2}\right)$ lies well outside of the bulk of the distribution of $\Delta$. To quantify this we will find the upper and lower probability of the complement of the smallest ball centered on the point estimator $\widehat{\mathbf{p}}=\left(\frac{z_{11}+k^{-2}}{n+1}, \ldots, \frac{z_{k k}+k^{-2}}{n+1}\right)$ and containing the null-hypothesis point $\left(k^{-2}, \ldots, k^{-2}\right)$.

To this end we generate $m$ samples $\left\{\Delta_{1}, \ldots \Delta_{m}\right\}$ from either the simplex or Dirichlet DS. Then, we compute the upper and lower distances $\mathcal{U}=\left\{u_{1}, \ldots u_{m}\right\}, \mathcal{L}=\left\{l_{1}, \ldots l_{m}\right\}$, where

$$
l_{i}=\inf \left\{\|\mathbf{y}-\widehat{\mathbf{p}}\|_{2}: \mathbf{y} \in \Delta_{i}\right\}, \quad u_{i}=\sup \left\{\|\mathbf{y}-\widehat{\mathbf{p}}\|_{2}: \mathbf{y} \in \Delta_{i}\right\}
$$

Leveraging the convexity of $\Delta_{i}, u_{i}$ can be computed as $u_{i}=\max \left\{\|\mathbf{e}-\widehat{\mathbf{p}}\|_{2}: \mathbf{e} \in V\left(\Delta_{i}\right)\right\}$, where $V\left(\Delta_{i}\right)$ is the set of vertexes of the convex polytope $\Delta_{i}$, and $l_{i}$ can be found by solving a convex optimization problem. We then use $u_{i}$ and $l_{i}$ and the distance $r_{\text {center }}=\left\|\widehat{\mathbf{p}}-\left(\frac{1}{k^{2}}, \ldots \frac{1}{k^{2}}\right)\right\|_{2}$ to define upper and lower $p$-values as

$$
\widehat{p}_{\text {upper }}\left(\left\{u_{1}, \ldots u_{m}\right\}\right)=\frac{\left|\mathcal{U} \geq r_{\text {center }}\right|}{m}, \quad \widehat{p}_{\text {lower }}\left(\left\{l_{1}, \ldots l_{m}\right\}\right)=\frac{\left|\mathcal{L} \geq r_{\text {center }}\right|}{m} .
$$

The upper $p$-value can be then used as usual $p$-value for testing the null hypothesis of uniformity at resolution $k$. The gap between the upper and lower $p$-value is specific to DS and measures lack of knowledge. In particular if the gap between upper and lower $p$-value indicates inability to make decision about independence at this resolution.

\section{Simulation Results}

To compare the performance of the Simplex and Dirichlet methods, we consider a simple test of independence. First, 100 data sets of sample size $n=30$ are generated under either the following null or alternate hypotheses:

$$
H_{0} \sim \operatorname{Beta}(1,1)^{2}, \quad H_{1} \sim \operatorname{Beta}(1,2)^{2} .
$$

Each of these data sets is then discretized into $2 \times 2,3 \times 3$, and $6 \times 6$ contingency tables and each table is tested for independence using the simplex DS, the Dirichlet DS, and the classical $\chi^{2}$ tests. To generate the $p$-values for the test of independence, both the simplex and Dirichlet DS generate 200 polytopes with a burn-in of 300 for the former. The purpose of the low sample size $(n=30)$ in this simulation is to demonstrate that as the resolution $k$ and the number of multinomial categories $k^{2}$ increases with sample size held constant, the uncertainty indicated between the gap between the upper and lower $p$-values increases. 

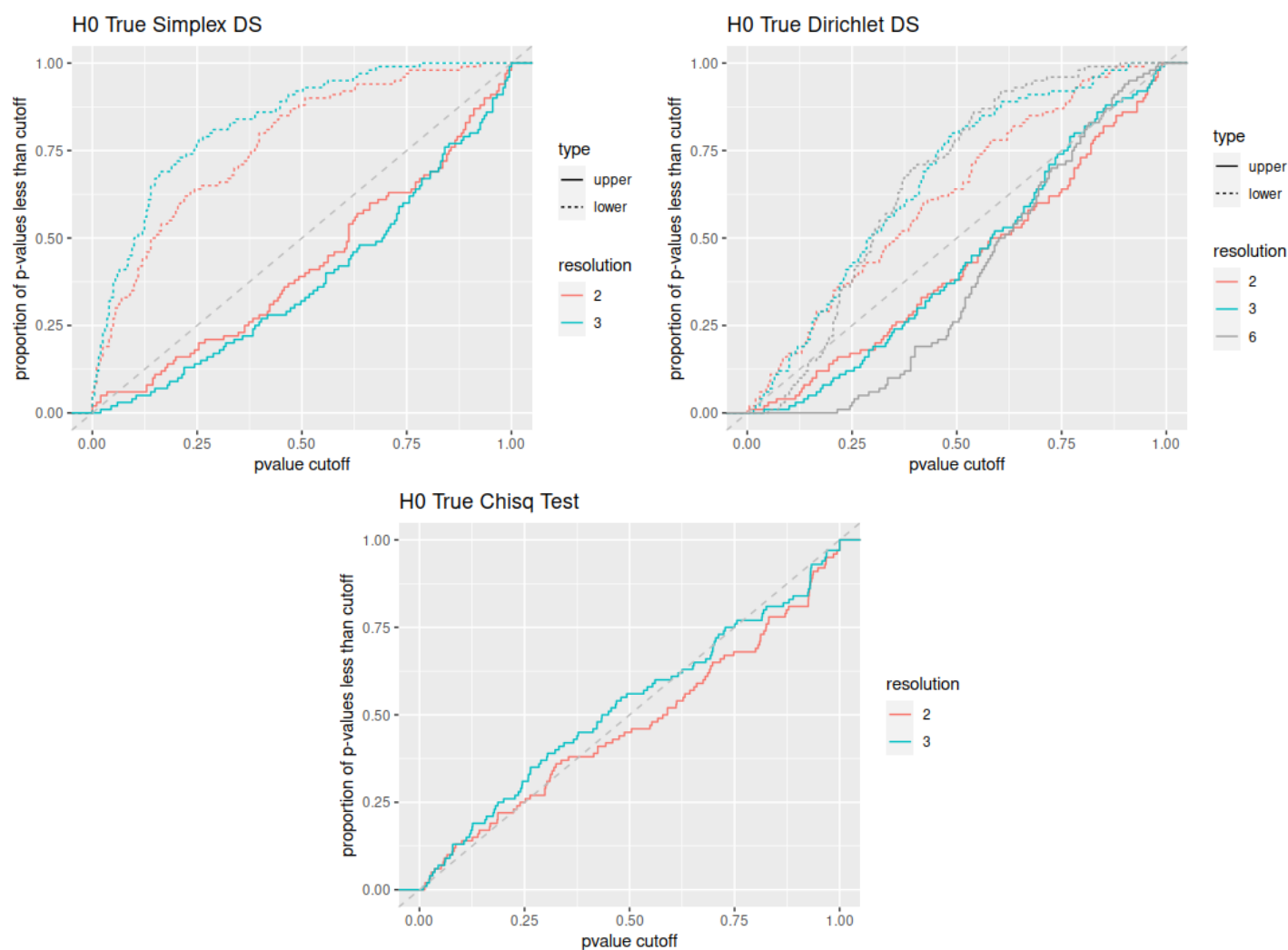

Figure 2: Empirical CDFs of the upper and lower $p$-values for $H_{0}$ analyzed using three tests: (Top Left): Simplex DS, (Top Right) Dirichlet DS, and (Bottom): $\chi^{2}$. The $x$-axis is the nominal $p$-value, the $y$-axis is proportion of $p$-values below $p$-value cutoff.

In Figure 2 we present plots of the Empirical CDFs of the upper and lower $p$-values under the assumption that $H_{0}$ is true. Well calibrated $p$-values follow a uniform distribution which CDF is represented by the $45^{\circ}$ line. As expected, we see that $p$-values empirical CDFs from the $\chi^{2}$ test closely follow this dotted line. The upper $p$-values for both DS tests are below the dotted line, showing that these $p$-values are conservative, i.e., sub-uniform. Next, we see that while the upper $p$-values for the Dirichlet and simplex method behave similarly, the lower $p$-values of the simplex method are more skewed towards rejecting. Consequently, Dirichlet DS has a much smaller gap between the upper and lower $p$-values than the simplex DS. Finally, we remark that there are no $p$-values for the $6 \mathrm{x} 6$ simplex method as the computation timed out after $2 \mathrm{hr}$ without producing a simplex. 

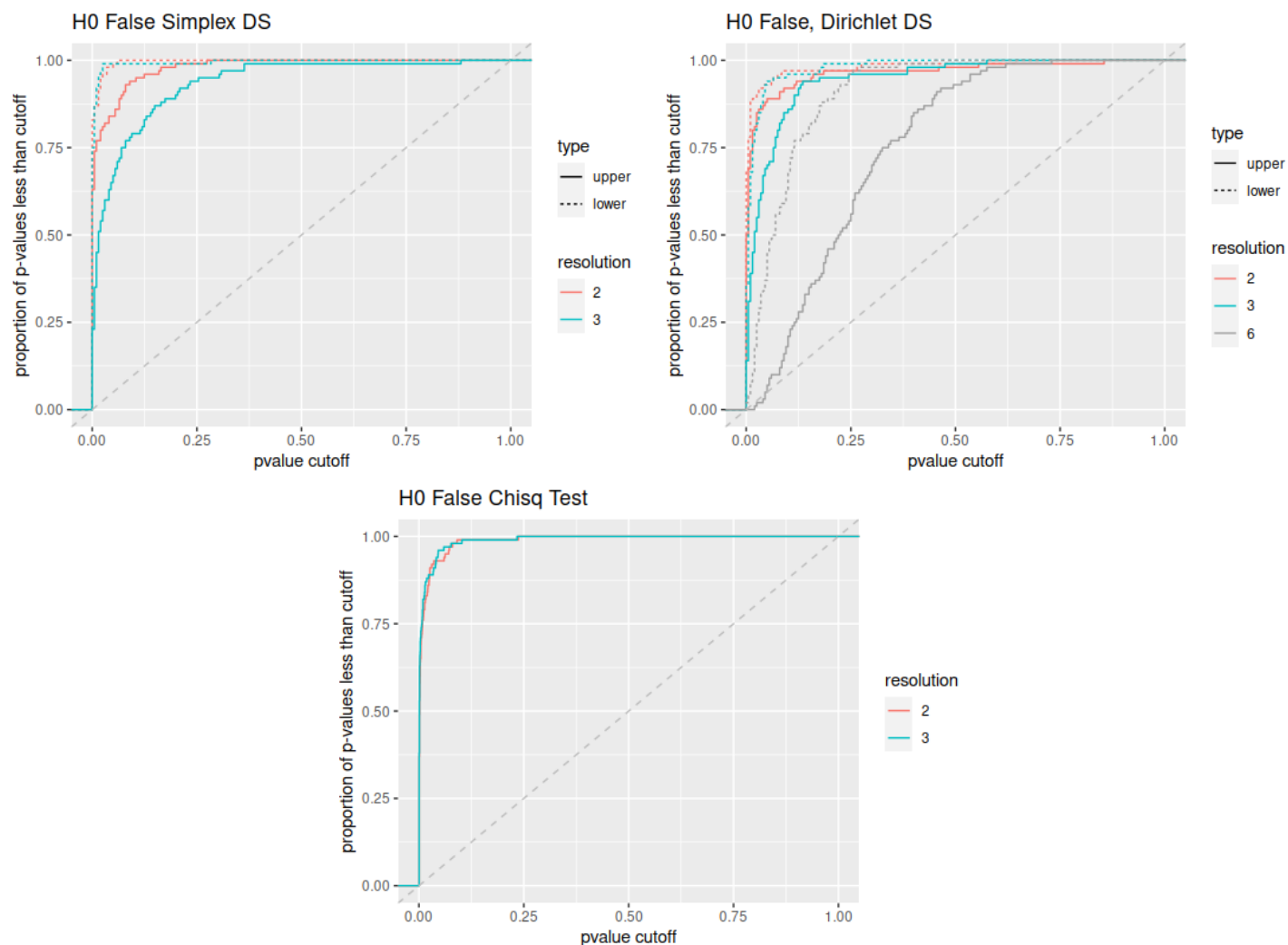

Figure 3: Empirical CDFs of the upper and lower $p$-values for $H_{0}$ analyzed using three tests: (Top Left): Simplex DS, (Top Right) Dirichlet DS, and (Bottom): $\chi^{2}$. The $x$-axis is the nominal $p$-value, the $y$-axis is proportion of $p$-values below the cutoff.

In Figure 3 we show empirical CDFs based on data generated under the alternate hypothesis in (4.1). All three tests correctly lean towards rejecting the null hypothesis. In terms of the power of the lower $p$-value, the simplex method performs similarly to the Dirichlet method. However, the empirical CDFs of upper $p$-values for the simplex method is lower than their corresponding empirical CDFs for the Dirichlet DS empirical Cr. This indicates that for the Dirichlet method has more power to reject $H_{0}$. In addition, we can see the gaps between lower and upper $p$-value plots increase as the resolution increases in both the simplex and Dirichlet DS.

As for runtime comparisons, the difference is substantial. Generating one polytope under Dirichlet DS at the $3 \times 3$ level takes approximately 2 seconds while a similar polytope takes nearly 30 seconds under Simplex DS. The difference in runtime comparison gets larger with the $6 \times 6$ level, where the Dirichlet DS is still under 5 seconds while the Simplex DS timed out after at least 2 hours. 


\begin{tabular}{|c|c|}
\hline Method & Runtime to generate one polytope \\
\hline Dirichlet Method for $2 \times 2$ & 1.76 seconds \\
\hline Dirichlet Method for $3 \times 3$ & 2.15 seconds \\
\hline Dirichlet Method for $6 \times 6$ & 4.15 seconds \\
\hline Simplex Method $2 \times 2$ & 5.31 seconds \\
\hline Simplex Method $3 \times 3$ & 29.60 seconds \\
\hline Simplex Method $6 \times 6$ & Timed out at $>2 \mathrm{hr}$ \\
\hline
\end{tabular}

\section{Discussion}

Based on this small simulation example, it appears that the Dirichlet DS might be better suited for performing the test for independence than the simplex DS both in terms of speed and frequentist performance. Dirichlet DS also has a relatively easy way to adapt to situations with missing data with a potential to be applied to adversarial attack scenarios. The one negative is that unlike simplex DS, the Dirichlet DS does not appear to be fiducial, i.e., based on an inverse of a data generating algorithm. On balance, it is not clear to us in what practical situations the simplex DS would be the preferable choice over the Dirichlet DS when conducting statistical inference.

\section{Acknowledgements}

Jan Hannig's research was supported in part by the National Science Foundation under Grant No. IIS-1633074 and DMS-1916115. Kai Zhang's research was supported in part by the National Science Foundation under Grant No. DMS-1613112, IIS-1633212, and DMS-1916237.

\section{References}

Chatterjee, S. (2020). A new coefficient of correlation. Journal of the American Statistical Association, in press.

Dempster, A. P. et al. (1966). New methods for reasoning towards posterior distributions based on sample data. The Annals of Mathematical Statistics 37(2), 355-374.

Gorsky, S. and L. Ma (2018). Multiscale Fisher's independence test for multivariate dependence. arXiv preprint arXiv:1806.06777.

Gretton, A., K. Fukumizu, C. Teo, L. Song, B. Schölkopf, and A. Smola (2007). A kernel statistical test of independence. Nips 20,585-592.

Hannig, J., H. Iyer, R. C. S. Lai, and T. C. M. Lee (2016). Generalized fiducial inference: A review and new results. Journal of the American Statistical Association 111(515), 1346-1361.

Heller, R., Y. Heller, and M. Gorfine (2013). A consistent multivariate test of association based on ranks of distances. Biometrika 100(2), 503-510.

Hoeffding, W. (1948). A non-parametric test of independence. The Annals of Mathematical Statistics, 546-557. 
Lawrence, E. C., S. Vander Wiel, C. Liu, and J. Zhang (2009). A new method for multinomial inference using Dempster-Shafer theory. Technical report, Los Alamos National Lab.(LANL), Los Alamos, NM (United States).

Lee, D., K. Zhang, and M. R. Kosorok (2019). Testing independence with the binary expansion randomized ensemble test. arXiv preprint arXiv:1912.03662.

Ma, L. and J. Mao (2019). Fisher exact scanning for dependency. Journal of the American Statistical Association 114 (525), 245-258.

Reshef, D. N., Y. A. Reshef, H. K. Finucane, S. R. Grossman, G. McVean, P. J. Turnbaugh, E. S. Lander, M. Mitzenmacher, and P. C. Sabeti (2011). Detecting novel associations in large data sets. Science 334(6062), 1518-1524.

Shi, H., M. Hallin, M. Drton, and F. Han (2020). Rate-optimality of consistent distribution-free tests of independence based on center-outward ranks and signs. arXiv preprint arXiv:2007.02186.

Székely, G. J., M. L. Rizzo, and N. K. Bakirov (2007, 12). Measuring and testing dependence by correlation of distances. The Annals of Statistics 35(6), 2769-2794.

Zhang, K. (2019). BET on independence. Journal of the American Statistical Association 114(528), $1620-1637$.

Zhang, K., Z. Zhao, and W. Zhou (2021). BEAUTY powered BEAST. arXiv preprint arXiv:2103.00674. 